\title{
ORIENTED MANIFOLDS THAT FIBER OVER $S^{4}$ \\ BY
}

STEVEN M. KAHN ${ }^{1}$

\begin{abstract}
Necessary and sufficient conditions are given for an oriented manifold $M$ to fiber up to cobordism over the 4-sphere $S^{4}$ (i.e. for $M$ to be oriented cobordant to a fiber bundle over $S^{4}$ ). The result here extends those previously obtained for fiberings over $S^{1}$ and $S^{2}$.

In addition, fiberings over products of surfaces are studied with complete solutions (in the sense above) being given in most cases including those of $S^{2} \times S^{2}$ and $\left(S^{1}\right)^{4}$.
\end{abstract}

1. Introduction. In this paper we completely determine the ideal of oriented cobordism classes $\omega \in \Omega_{*}$ that fiber (i.e. that are represented by a fiber bundle) over the 4-sphere $S^{4}$.

Our result here extends the results of Burdick [5], Neumann [14], Conner [7] and those of [1] concerning bundles over $S^{1}$ and $S^{2}$. Burdick, who was the first to consider this type of problem in the context of oriented cobordism (the general problem was originally introduced by Conner and Floyd [8]), determined the fibering ideal of $S^{1}$ up to the torsion in $\Omega_{*}$. Conner subsequently determined the fibering ideal of $S^{2}$ again modulo the torsion in $\Omega_{*}$. The complete solutions were given by Neumann and in [1], respectively.

A class $\omega \in \Omega_{n}$ fibers over the circle if and only if the signature $\sigma(\omega)$ is zero.

A class $\omega \in \Omega_{n}$ fibers over $S^{2}$ if and only if $\sigma(\omega)=0$ if $n$ is even and if and only if the Stiefel-Whitney number $w_{2} w_{n-2}(\omega)$ (the so-called De Rham invariant) is zero if $n$ is odd.

Examples of applications of these and similar results can be found in the works of Kreck [12] and Chess [6].

In a related study, we also consider fibrations over products of surfaces. Complete solutions are obtained in all cases except where both surfaces have negative Euler characteristic. In particular, the fibering ideals of $S^{2} \times S^{2}, \mathbf{R} P(2) \times \mathbf{R} P(2)$ and $\left(S^{1}\right)^{4}$ for example, are found.

We now state our main result.

Let $w_{i}(M), v_{i}(M)$ and $p_{i}(M)$ denote the $i$ th Stiefel-Whitney, Wu and Pontrjagin class of $M$, respectively. Let $\sigma(M)$ denote the signature.

Received by the editors August 29, 1983 and, in revised form, January 3, 1984.

1980 Mathematics Subject Classification. Primary 57R75, 57R20, 55R10.

Key words and phrases. Oriented cobordism class, fibration, Stiefel-Whitney numbers, Wu class, Wall's ring.

${ }^{1}$ This research was partially supported by a Grant-in-Aid from Wayne State University. 
THEOREM 1. A class $\omega \in \Omega_{n}(n \geqslant 4)$ fibers over $S^{4}$ if and only if

(i) $\sigma(\omega)=0$ and $p_{1}^{2}(\omega) \equiv 0(\bmod 3)$ if $n=8$,

(ii) $\sigma(\omega)=0$ and $\mathrm{Sq}^{2} \mathrm{Sq}^{1} v_{2 k-2} \mathrm{Sq}^{1} v_{2 k-2}(\omega)=0$ if $n=4 k \neq 8$,

(iii) $w_{2} w_{4 k-1}(\omega)=0$ if $n=4 k+1$,

(iv) $w_{4} w_{4 k-2}(\omega)=0$ if $n=4 k+2$,

(v) $w_{2}^{3} \mathrm{Sq}^{2 k-3} v_{2 k}(\omega)=0$ and $w_{6} \mathrm{Sq}^{2 k-3} v_{2 k}(\omega)=0$ if $n=4 k+3$.

There is a pattern to these obstructions that can be seen in a slightly different, more general statement of Theorem 1. Accordingly, let $\tau_{M}: M^{n} \rightarrow$ BSO classify the stable tangent bundle of the orientable manifold $M^{n}$ and let

$$
I_{n}(\mathrm{SO}, 2)=\bigcap_{M^{n}} \operatorname{Ker} \tau_{M}^{*}
$$

denote the Brown-Peterson relations for orientable $n$-manifolds. Following the notation used in [9], let

$$
I_{\mathrm{SO}}^{k}\left(M^{n}\right)=\left\{\alpha \in H^{k}(\mathrm{BSO}) \mid \text { if } k \leqslant n, \tau_{M}^{*}(\alpha \beta)=0 \forall \beta \in H^{n-k}(\mathrm{BSO})\right\}
$$

and let

$$
A_{\text {SO }}^{*}\left(M^{n}\right)=H^{*}(\mathrm{BSO}) / I_{\mathrm{SO}}^{*}\left(M^{n}\right) .
$$

We can rewrite Theorem 1 as follows:

(1.1) A class $\omega \in \Omega_{n}$ fibers over $S^{4}$ if and only if

(a) $\sigma(\omega)=0$,

(b) $\left[\left(I_{n-4}(\mathrm{SO}, 2)\right)^{2}\right]=0 \in A_{\mathrm{SO}}^{*}(\omega)$,

(c) $\left[\operatorname{Sq}^{r}\left(I_{n-4}^{q}(\mathrm{SO}, 2)\right)\right]=0 \in A_{\mathrm{SO}}^{*}(\omega)$ for $r>q-4$, and

(d) $p_{1}^{2}(\omega) \equiv 0(\bmod 3)$ if $n=8$.

REMARK 1.2. That Theorem 1 and (1.1) are equivalent is seen as follows: By the $\mathrm{Wu}$ formula and the fact that the odd $\mathrm{Wu}$ classes of an orientable manifold vanish, we have

$$
\begin{aligned}
& w_{4 k-1}\left(M^{4 k+1}\right)=\mathrm{Sq}^{2 k-2} v_{2 k}(M) \in \tau_{M}^{*} \operatorname{Sq}^{2 k-1}\left(I_{4 k-3}^{2 k}(\mathrm{SO}, 2)\right), \\
& w_{4 k-2}\left(M^{4 k+2}\right)=\mathrm{Sq}^{2 k-2} v_{2 k}(M) \in \tau_{M}^{*} \mathrm{Sq}^{2 k-2}\left(I_{4 k-2}^{2 k}(\mathrm{SO}, 2)\right), \\
& \mathrm{Sq}^{2 k-3} v_{2 k}\left(M^{4 k+3}\right) \in \tau_{M}^{*} \mathrm{Sq}^{2 k-3}\left(I_{4 k-1}^{2 k}(\mathrm{SO}, 2)\right) .
\end{aligned}
$$

In addition, it will be shown later (in the proof of Theorem 1) that $\mathrm{Sq}^{1} v_{2 k-2}$ and therefore $\mathrm{Sq}^{2} \mathrm{Sq}^{1} v_{2 k-2}$ belong to $I_{4 k-4}(\mathrm{SO}, 2)$. Consequently, proving the necessity of the conditions listed in (1.1) proves the necessity of the conditions given in Theorem 1. Proving the sufficiency of the conditions given in Theorem 1 proves the sufficiency of those listed in (1.1). This is the approach we will take.

REMARK 1.3. It is interesting to note that for unoriented fiberings over $S^{4}$, conditions (b) and (c) of (1.1) (with SO replaced by $\mathrm{O}$ ) are actually equivalent obstructions [11, Proposition 2.4]. This is not true in the oriented case. Indeed let $X_{5}$ and $X_{11}$ denote the generators of $\Omega_{5}$ and $\Omega_{11}$, respectively. Combining Brown-Peterson's computation of $I_{n}(\mathrm{SO}, 2)$ [3, Theorems 4.3 and 4.4] with a calculation of the Stiefel-Whitney numbers of $X_{5} X_{11}$, one sees that

$$
\left[\mathrm{Sq}^{r}\left(I_{12}^{q}(\mathrm{SO}, 2)\right)\right]=0 \in A_{\mathrm{SO}}^{*}\left(X_{5} X_{11}\right) \text { for } r>q-4 \text {. }
$$


But $\left[\left(I_{12}(\mathrm{SO}, 2)\right)^{2}\right] \neq 0 \in A_{\text {SO }}^{*}\left(X_{5} X_{11}\right)$. (A routine calculation shows that

$$
\left.\mathrm{Sq}^{2} \mathrm{Sq}^{1} v_{6} \cdot \mathrm{Sq}^{1} v_{6}\left(X_{5} X_{11}\right) \neq 0 .\right)
$$

REMARK 1.4. The condition that $\mathrm{Sq}^{2} \mathrm{Sq}^{1} v_{2 k-2} \mathrm{Sq}^{1} v_{2 k-2}\left(M^{4 k}\right)=0$ is a "new" obstruction to fibering a manifold over $S^{4}$. It is not one that appears in either the unoriented or complex case. This distinguishes the $S^{4}$ solution from those corresponding to $S^{1}$ and $S^{2}$. It corresponds to the fact that although there exists a manifold fibered over $S^{4}$ that is cobordant to $X_{5} X_{11}$, there does not exist any such orientable manifold.

THEOREM 2. Let $B_{1}$ be any surface and let $B_{2}$ be any surface with $\chi\left(B_{2}\right) \geqslant 0$. A class $\omega \in \Omega_{n}(n>5)$ fibers over $B_{1} \times B_{2}$ if and only if

(i) $\sigma(\omega)=0$

and, if either $\chi\left(B_{1}\right)$ or $\chi\left(B_{2}\right)$ is even and $n$ is odd,

(ii) $w_{2} w_{n-2}(\omega)=0$.

Question. If $B_{1}$ and $B_{2}$ are both surfaces with negative Euler characteristic, then any oriented manifold fibered over $B_{1} \times B_{2}$ has signature divisible by four [13]. But is there a manifold $M$ fibered over $B_{1} \times B_{2}$ with $\sigma(M)=4$ ?

The proof of Theorem 2 is given in $\S 4$. The remainder of the paper is devoted to the proof of Theorem 1. As one can guess from the history of the problem, the key here, aside of course from determining the actual characteristic number obstructions, is getting some sort of handle on the torsion in $\Omega_{*}$. This is done in $\S 2$ and constitutes the bulk of the proof of sufficiency. In $\$ 3$, necessity is shown and the proof of Theorem 1 is formally completed.

I would like to thank the referee for his helpful suggestions.

2. An analysis of Tor $\Omega_{*}$. The main problem in dealing with the oriented cobordism ring is its structure. More to the point, the structure of the torsion in $\Omega_{*}$ is what is so difficult to handle. Accordingly, the purpose of this section is to obtain a manageable description of Tor $\Omega_{*}$ in terms of the fibering ideal of $S^{4}$. This is done through the following

THEOREM 2.1. Let $\omega \in$ Tor $\Omega_{n}$. Then

$$
\omega= \begin{cases}N X_{5} X_{11}+\text { Fib. } & \text { if } n=4 k, \\ N X_{5}+\text { Fib. } & \text { if } n=4 k+1, \\ N X_{5}^{2}+\text { Fib. } & \text { if } n=4 k+2, \\ N_{1} X_{11}+N_{2} X_{5}^{3}+\text { Fib. } & \text { if } n=4 k+3\end{cases}
$$

where Fib. denotes some class fibered over $S^{4}$ and $N$ denotes some class of the form $\sum \Pi \mathrm{C} P\left(2^{j}\right), j \geqslant 0$, or the one-point space.

The rest of this section constitutes the proof of this theorem. The notation used above (i.e. $N$ and Fib.) will be used throughout, while no notation will be used to distinguish between a manifold and its cobordism class. In addition, let $\delta(M)$ be the 
line bundle over the manifold $M$ with $w_{1}(\delta(M))=w_{1}(M)$. We will denote by $\mathbf{R} \boldsymbol{P} \delta\left(q_{n}, \ldots, q_{1}, M\right)$ the iterated projective bundle

$$
\begin{gathered}
\mathbf{R} P\left(\delta_{n} \underset{\oplus}{\oplus} \theta^{q_{n}}\right) \\
\vdots \\
\vdots \\
\vdots \\
\mathbf{R} P\left(\delta_{1} \stackrel{\oplus}{\oplus} \theta^{q_{1}}\right) \\
\vdots \\
M
\end{gathered}
$$

where $\delta_{1}=\delta(M), \delta_{i}=\delta\left(\mathbf{R} P\left(\delta_{i-1} \oplus \theta^{q_{1-1}}\right)\right)$ and $\theta^{j}$ denotes the trivial $j$-bundle.

Proof of TheOrem 2.1. Our approach is through Wall's ring $\mathscr{W}_{*}$ which consists of those classes in the unoriented cobordism ring $\mathfrak{N}_{*}$ that have a representative $W$ with $w_{1}^{2}(W)=0$. In [17] Wall showed that every class in Tor $\Omega_{n}$ lies in the image of the map $\partial: \mathscr{W}_{n+1} \rightarrow \Omega_{n}$ which assigns to $M$ the oriented class of the submanifold of $M$ dual to $w_{1}(M)$. Our take-off point is a bit further down the line. In [2] Anderson, using Wall's result, showed that every class in Tor $\Omega_{n}$ could be represented as a sum of manifolds of the form $\mathbf{R} P \delta(2 j+1, M)$. If we appeal to his proof we get an even sharper description. Namely,

(2.2) (Anderson). Let $\omega \in \operatorname{Tor} \Omega_{n}$. Then $\omega=\sum \mathbf{R} P \delta\left(2^{r+2} s-1,2^{r+1}, W\right)$, where $W \in \mathscr{W}_{*}$ and $r \geqslant 0, s>0$.

The proof of Theorem 2.1 then comes down to two things: an analysis of $\mathscr{W}_{*}$ in terms of the fibering ideal of $S^{4}$ and an analysis of the homomorphism

$$
\mathbf{R} \boldsymbol{P} \delta\left(2^{r+2} s-1,2^{r+1},-\right): \mathscr{W}_{*} \rightarrow \text { Tor } \Omega_{*} \text {. }
$$

(Note that $\mathbf{R} P \delta(2 j+1, M)$ is orientable and its oriented cobordism class depends only on the class of $M$ in $\mathfrak{N}_{*}$.)

First, $\mathscr{W}_{*}$ is a polynomial algebra over $Z_{2}$ with one generator $Y_{i}$ in each dimension $i$ except those of the form $2^{t}-1$ and $i=2$. Choosing these generators we recall that for $i \neq 2,4,5,6$ or 11 there are generators $X_{i}$ of $\mathfrak{N}_{i}$ that fiber over $S^{4}$ [15]. (For future use we let $X_{4}, X_{6}$ and $X_{11}$ denote Brown's generators [4] fibered over $S^{2}$.) We let $Y_{2 l+1}=X_{2 l+1}$ (these are orientable) and we let $Y_{2^{j+1}}=\mathbf{C P}\left(2^{j}\right)$. For even $i \neq 2^{j+1}$ we choose Anderson's generators. Specifically, let $Y_{2 k}, k=2^{r}(2 s+1)$ with $s>0$, be the manifold $M\left(2^{r+2} s-1,2^{r+1}, 1\right)$, where

$$
\begin{aligned}
& M(m, n, 1)=\mathbf{R} P\left(\eta \oplus \theta^{m}\right) \\
& \downarrow \\
& \mathbf{R} P\left(\lambda \oplus \theta^{n}\right) \\
& \downarrow \\
& S^{1}
\end{aligned}
$$

with $\eta$ and $\lambda$ being the appropriate canonical line bundles. Let $Y_{0}=$ one-point space.

Before proceeding, we might point out that there are standard formulas for computing the characteristic classes of projective bundles. Because of the many papers that describe these calculations (e.g. see $[4,8,11])$, here we will omit such details. 
LEMMA 2.3. The unoriented cobordism class of $Y_{2 k}$ is given by

$$
Y_{2 k}= \begin{cases}a X_{2}^{k-3}\left(X_{2} X_{4}+X_{6}\right)+b X_{2}^{k-5}\left(X_{4} X_{6}+X_{5}^{2}\right)+\text { Fib. } & \text { if } k \text { is odd, } \\ a X_{2}^{k-5}\left(X_{4} X_{6}+X_{5}^{2}\right)+\text { Fib. } & \text { if } k \text { is even, }\end{cases}
$$

where $a, b \in \mathbf{Z}_{2}$ and $X_{2}=\mathbf{R} P(2)$.

Proof. The ideal of unoriented cobordism classes that fiber over $S^{4}$ is for the most part known [11]. In particular, $Y_{2 k}$ (indeed any class in $\mathfrak{R}_{2 k}$ ) can be written as

$$
Y_{2 k}=a_{1} X_{2}^{k}+a_{2} X_{2}^{k-3} X_{2} X_{4}+a_{3} X_{2}^{k-3} X_{6}+a_{4} X_{2}^{k-5} X_{4} X_{6}+a_{5} X_{2}^{k-5} X_{5}^{2}+\text { Fib., }
$$

and since $w_{2 k}\left(Y_{2 k}\right)=0$ we further know that $a_{1}=0$. In [16, Proposition 7.2], Stong showed that the classes $X_{2}^{k-3} X_{2} X_{4}$ and $X_{2}^{k-3} X_{6}$ are distinguished by those StiefelWhitney numbers divisible by $w_{2 k}, w_{2 k-1}, w_{2 k-2}$ or $w_{2 k-3}$. In addition, by [15, Proposition 1.1] any such number vanishes on any class that fibers over $S^{4}$. In the case where $k$ is even, a projective bundle computation shows that these same Stiefel-Whitney numbers of $Y_{2 k}$ are zero. Hence $a_{2}=a_{3}=0$. For $k$ odd, we use the fact that

$$
w_{1}^{3} w_{2 k-3}\left(X_{2}^{k-3} X_{2} X_{4}\right)=w_{1}^{3} w_{2 k-3}\left(X_{2}^{k-3} X_{6}\right)=1
$$

while

$$
w_{1}^{3} w_{2 k-3}\left(X_{2}^{k-5} X_{4} X_{6}\right)=w_{1}^{3} w_{2 k-3}\left(X_{2}^{k-5} X_{5}^{2}\right)=0 .
$$

Since $Y_{2 k} \in \mathscr{W}_{2 k}, w_{1}^{3} w_{2 k-3}\left(Y_{2 k}\right)=0$ and it follows that $a_{2}=a_{3}$.

It is left to show that $a_{4}=a_{5}$. Appealing to our study in [11] we note that the characteristic number $v_{4} \mathrm{Sq}^{k-3} v_{k-1}$ is nonzero on the classes $X_{2}^{k-5} X_{4} X_{6}$ and $X_{2}^{k-5} X_{5}^{2}$ while zero on the classes $X_{2}^{k-3} X_{2} X_{4}$ and $X_{2}^{k-3} X_{6}$. It is also zero on any class fibered over $S^{4}$ [11, Theorem 1]. The result then follows from the following

Claim. $v_{4} \mathrm{Sq}^{k-3} v_{k-1}\left(Y_{2 k}\right)=0$.

Proof of Claim. (i) Let $k$ be odd. By the Wu formula,

$$
v_{4} \mathrm{Sq}^{k-3} v_{k-1}\left(Y_{2 k}\right)=\left(v_{4} w_{2 k-4}+v_{4} \mathrm{Sq}^{k-4} v_{k}+v_{4} \mathrm{Sq}^{k-2} v_{k-2}\right)\left(Y_{2 k}\right) \text {. }
$$

Now

$$
v_{4} \mathrm{Sq}^{k-2} v_{k-2}=\mathrm{Sq}^{4}\left(v_{k-2}^{2}\right)=\mathrm{Sq}^{k} \mathrm{Sq}^{2} v_{k-2}=v_{k} \mathrm{Sq}^{2} v_{k-2}
$$

and since $k$ is odd, $v_{k}$ and $v_{k-2}$, and so $\mathrm{Sq}^{2} v_{k-2}$, are multiples of $w_{1}$. Thus $v_{4} \mathrm{Sq}^{k-2} v_{k-2}$ is a multiple of $w_{1}^{2}$ and so vanishes on $Y_{2 k}$. Next, using the Adem relations, the $\mathrm{Wu}$ formula and again the fact that $v_{\text {odd }}$ is a multiple of $w_{1}$, we have that when evaluated on $Y_{2 k}$,

$$
\begin{aligned}
v_{4} \mathrm{Sq}^{k-4} v_{k} & =\mathrm{Sq}^{4} \mathrm{Sq}^{k-4} v_{k}=\left(\begin{array}{c}
k-6 \\
2
\end{array}\right) \mathrm{Sq}^{k-1} \mathrm{Sq}^{1} v_{k} \\
& =\left(\begin{array}{c}
k-6 \\
2
\end{array}\right) \mathrm{Sq}^{3} \mathrm{Sq}^{k-2} v_{k}=\left(\begin{array}{c}
k-6 \\
2
\end{array}\right) v_{3} w_{2 k-3}
\end{aligned}
$$


Hence

$$
v_{4} \mathrm{Sq}^{k-3} v_{k-1}\left(Y_{2 k}\right)=\left(\left(\begin{array}{c}
k-6 \\
2
\end{array}\right) v_{3} w_{2 k-3}+v_{4} w_{2 k-4}\right)\left(Y_{2 k}\right)
$$

A standard computation shows that $v_{3} w_{2 k-3}\left(Y_{2 k}\right)=1$ and $v_{4} w_{2 k-4}\left(Y_{2 k}\right)=1$ or 0 depending on whether $k=4 l+1$ or $k=4 l+3$, respectively. Thus

$$
v_{4} \mathrm{Sq}^{k-3} v_{k-1}\left(Y_{2 k}\right)=0
$$

(ii) Let $k$ be even. Using arguments as before, when evaluated on $Y_{2 k}$,

$v_{4} \mathrm{Sq}^{k-3} v_{k-1}=\mathrm{Sq}^{4} \mathrm{Sq}^{k-3} v_{k-1}=\left(\begin{array}{c}k-5 \\ 2\end{array}\right) \mathrm{Sq}^{k} \mathrm{Sq}^{1} v_{k-1}=\left(\begin{array}{c}k-5 \\ 2\end{array}\right)\left(\mathrm{Sq}^{1} v_{k-1}\right)^{2}=0$.

We now have the following description of $\mathscr{W}_{*}$.

Corollary 2.5. Let $W \in \mathscr{W}_{n}$. Then $W=\sum N X_{5}^{q} X_{11}^{t} Y_{2 k}+$ Fib., where $q, t, k \geqslant 0$.

Proof. Appealing to the structure of $\mathscr{W}_{*}$ as a polynomial algebra, we have $W=\sum \Pi C P\left(2^{j}\right) \Pi X_{2 l+1} \Pi Y_{2 k}$. But by Lemma 2.3 and the fact that each of the unoriented classes $X_{4}^{2}, X_{6}^{2}, X_{4} X_{5}^{2}$ and $X_{6} X_{5}^{2}$ fibers over $S^{4}$ (see [4 and 11]), any product of $Y_{2 k}$ 's fibers over $S^{4}$. The result follows when one recalls that $X_{2 l+1}$ fibers over $S^{4}$ for all $2 l+1 \neq 5$ or 11 .

Now we turn to the homomorphism $\mathbf{R} P \delta\left(2^{r+2} s-1,2^{r+1},-\right)$ and present a few simple but quite key observations concerning it. We begin with the triviality

$$
\mathbf{R} P \delta(q, \text { Fib. })=\text { Fib. }
$$

Next, given two manifolds $M$ and $N$ with $N$ orientable, $\delta(M \times N)=p^{*} \delta(M)$, where $p: M \times N \rightarrow M$ is the projection map. Thus

$$
\mathbf{R} P \delta(q, M \times N)=N \times \mathbf{R} P \delta(q, M) \text { if } N \text { is orientable. }
$$

Finally, Anderson calculated that

$$
\partial\left(\prod_{j=1}^{t} M\left(m_{j}, n_{j}, 1\right)\right)=\mathbf{R} P \delta\left(m_{t}, n_{t}, \prod_{j=1}^{t-1} M\left(m_{j}, n_{j}, 1\right)\right) .
$$

By considering the case where $t=2$, we get

(2.8) $\mathbf{R} \boldsymbol{P} \boldsymbol{\delta}\left(m_{2}, n_{2}, Y_{2 k_{1}}\right)=\mathbf{R} \boldsymbol{P} \boldsymbol{\delta}\left(m_{1}, n_{1}, Y_{2 k_{2}}\right)$, where $Y_{2 k_{1}}=M\left(m_{i}, n_{i}, 1\right)$,

and by recalling that $\rho \circ \partial$ is a derivation, where $\rho: \Omega_{*} \rightarrow \mathscr{W}_{*}$ is the forgetful map, we also get

$$
\mathbf{R} P \delta\left(m, n, Y_{2 k}\right)=0 \text { for } Y_{2 k}=M(m, n, 1) .
$$

The observations of (2.6) and (2.7) combined with Corollary 2.5 give an immediate refinement of Anderson's Theorem.

COROllary 2.10. Let $\omega \in$ Tor $\Omega_{n}$. Then

$$
\omega=\sum N X_{5}^{q} X_{11}^{t} \mathbf{R} P\left(2^{r+2} s-1,2^{r+1}, Y_{2 k}\right)+\text { Fib., where } q, t, k \geqslant 0 \text {. }
$$

Clearly, except for a few loose ends, the proof of Theorem 2.1 reduces to the determination of $\mathbf{R} \boldsymbol{P} \boldsymbol{\delta}\left(2^{r+2} s-1,2^{r+1}, Y_{2 k}\right)$. The following extension of Lemma 2.3 makes this determination possible. 
LEMMA 2.11. (i) $Y_{6}=X_{2} X_{4}+X_{6}$.

(ii) $Y_{10}=X_{4} X_{6}+X_{5}^{2}+a X_{2}^{2}\left(X_{2} X_{4}+X_{6}\right)+$ Fib.

(iii) $Y_{12}=X_{2} X_{4} X_{6}+X_{2} X_{5}^{2}+$ Fib.

Proof. (i) This follows from the fact that $Y_{6}$ is indecomposable.

(ii) $Y_{10}=X_{10}+a X_{2}^{2}\left(X_{2} X_{4}+X_{6}\right)+b\left(X_{4} X_{6}+X_{5}^{2}\right)+c X_{8} X_{2}+d X_{4}^{2} X_{2}$. Computing $s$-numbers, we have $s_{10}\left(Y_{10}\right)=1$ and $s_{5,5}\left(Y_{10}\right)=0$. That $b=1$ follows from the fact [11, Lemma 4.7] that $s_{10}\left(X_{10}\right)=s_{5,5}\left(X_{10}\right)=1$.

(iii) $Y_{12}=X_{12}+a\left(X_{2} X_{4} X_{6}+X_{2} X_{5}^{2}\right)+b X_{10} X_{2}+$ (other decomposables). Computing $s$-numbers, $s_{12}\left(Y_{12}\right)=s_{10,2}\left(Y_{12}\right)=1$ while $s_{2,5,5}\left(Y_{12}\right)=0$. That $a=1$ follows from the fact [11, Lemma 4.9] that $s_{12}\left(X_{12}\right)=1, s_{10,2}\left(X_{12}\right)=s_{2,5,5}\left(X_{12}\right)=0$ and $s_{5,5}\left(X_{10}\right)=1$.

The key point here (referring back to Lemma 2.3) is that every Anderson generator $Y_{2 k}$ can actually be expressed simply in terms of $Y_{6}$ and $Y_{10}$, or $Y_{12}$. Specifically we have

COROllary 2.12 .

$$
Y_{2 k}= \begin{cases}a X_{2}^{k-3} Y_{6}+b X_{2}^{k-5} Y_{10}+\text { Fib. } & \text { if } k \text { is odd } \\ a X_{2}^{k-6} Y_{12}+\text { Fib. } & \text { if } k \text { is even. }\end{cases}
$$

With this reduction we prove

PROPOSITION 2.13. RP $\boldsymbol{P}\left(2^{r+2} s-1,2^{r+1}, Y_{2 k}\right)=\sum N X_{5}^{q} X_{11}^{t}+$ Fib.

Proof. (i) We first consider the case where $k>0$. By Corollary 2.12 and (2.7) (note that $X_{2}^{2}=\mathbf{C P}(2)$ ),

$$
\begin{aligned}
\mathbf{R} P \boldsymbol{\delta} & \left(2^{r+2} s-1,2^{r+1}, Y_{2 k}\right) \\
= & \left\{\begin{array}{cl}
a_{1} X_{2}^{k-3} \mathbf{R} P \delta\left(2^{r+2} s-1,2^{r+1}, Y_{6}\right) \\
+a_{2} X_{2}^{k-5} \mathbf{R} P \delta\left(2^{r+2} s-1,2^{r+1}, Y_{10}\right)+\text { Fib. } & \text { if } k \text { is odd, } \\
a X_{2}^{k-6} \mathbf{R} P \delta\left(2^{r+2} s-1,2^{r+1}, Y_{12}\right)+\text { Fib. } & \text { if } k \text { is even, }
\end{array}\right.
\end{aligned}
$$

and by (2.8),

$$
= \begin{cases}a_{1} X_{2}^{k-3} \mathbf{R} P \delta\left(3,2, Y_{2 l}\right)+a_{2} X_{2}^{k-5} \mathbf{R} P \delta\left(7,2, Y_{2 l}\right)+\text { Fib. } & \text { if } k \text { is odd } \\ a X_{2}^{k-6} \mathbf{R} P \delta\left(7,4, Y_{2 l}\right)+\text { Fib. } & \text { if } k \text { is even }\end{cases}
$$

where $Y_{2 l}=M\left(2^{r+2} s-1,2^{r+1}, 1\right)$.

If we invoke Corollary 2.12 and (2.7) again (this time applied to $Y_{2 l}$ ), we see that we need only consider the classes of $\mathbf{R} P \delta\left(p, q, Y_{2 k}\right)$, where $(p, q)=(3,2),(7,2)$ or $(7,4)$ and $2 k=6,10$ or 12 . By $(2.9)$ however, $\mathbf{R} P \delta\left(3,2, Y_{6}\right)=\mathbf{R} P \delta\left(7,2, Y_{10}\right)=$ $\mathbf{R} P \delta\left(7,4, Y_{12}\right)=0$. And by $(2.8), \mathbf{R} P \delta\left(7,4, Y_{6}\right)=\mathbf{R} P \delta\left(3,2, Y_{12}\right), \cdot \operatorname{R} P \delta\left(7,4, Y_{10}\right)=$ $\mathbf{R} \boldsymbol{P} \delta\left(7,2, Y_{12}\right)$ and $\mathbf{R} \boldsymbol{P} \delta\left(7,2, Y_{6}\right)=\mathbf{R} \boldsymbol{P} \delta\left(3,2, Y_{10}\right)$. So the problem comes down to computing (a) $\mathbf{R} P \delta\left(3,2, Y_{10}\right)$, (b) $\mathbf{R} P \delta\left(3,2, Y_{12}\right)$, and (c) $\mathbf{R} P \delta\left(7,2, Y_{12}\right)$. 
(a) Let

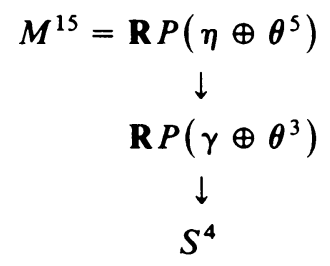

where $\gamma$ is the quaternionic line bundle over $S^{4}=\mathbf{H} P(1)$. A standard check of Stiefel-Whitney classes shows that $M^{15}$ is orientable $\left(w_{1}(M)=0\right)$ and $M^{15} \neq 0 \in$ $\Omega_{15}$. By Corollary $2.10, M^{15}=a_{1} \mathbf{C P}(2) X_{11}+a_{2} X_{5}^{3}+a_{3} \mathbf{R} P \delta\left(3,2, Y_{10}\right)$. But since $M^{15}$ fibers over $S^{4}, a_{3} \neq 0$ [11, Proposition 3.3]. Hence $\mathbf{R} P \delta\left(3,2, Y_{10}\right)=a_{1} \mathbf{C P}(2) X_{11}$ $+a_{2} X_{5}^{3}+$ Fib.

(b) $\mathbf{R} \boldsymbol{P} \delta\left(3,2, Y_{12}\right)=\mathbf{R} \boldsymbol{P} \boldsymbol{\delta}\left(3, Z^{14}\right)$, where $Z^{14}$ is some 14-manifold. If we express $Z$ as in (2.4) and then "factor out" $X_{2}^{2}$ using (2.7), we have $\mathbf{R} P \delta\left(3,2, Y_{12}\right)=$ $\mathbf{C} P(2) \mathbf{R} P \delta\left(3, Z^{10}\right)$, where $Z^{10}$ is some 10 -manifold. Now $\Omega_{13}$ is generated by $X_{13}$, $\mathbf{C P}(2) X_{9}, \mathbf{C P}(4) X_{5}$ and $\mathbf{C P}(2)^{2} X_{5}$. Therefore $\mathbf{R} P \delta\left(3, Z^{10}\right)=N X_{5}+$ Fib. and so $\mathbf{R} P \delta\left(3,2, Y_{12}\right)=N X_{5}+$ Fib., where as usual $N=\Sigma \Pi \mathbf{C} P\left(2^{j}\right), j \geqslant 0$.

(c) Arguing as in (b), we can write

$$
\mathbf{R} P \delta\left(7,2, Y_{12}\right)=a_{1} \mathbf{C P}(2)^{2} \mathbf{R} P \delta\left(7, Z^{6}\right)+a_{2} \mathbf{C P}(2) \mathbf{R} P \delta\left(7, X_{4} X_{6}\right)+\text { Fib. }
$$

An $s$-number computation in combination with (2.4) shows that $\mathbf{R} P \delta\left(4, Y_{6}\right)=X_{4} X_{6}$ $+a X_{5}^{2}+$ Fib. Thus

$$
\begin{aligned}
\mathbf{R} P \delta\left(7, X_{4} X_{6}\right) & =\mathbf{R} P \delta\left(7,4, Y_{6}\right)+\text { Fib. }=\mathbf{R} P \delta\left(3,2, Y_{12}\right)+\text { Fib. } \\
& =N X_{5}+\text { Fib. } \quad(\text { by (b) }) .
\end{aligned}
$$

Since $\mathbf{R} \boldsymbol{P} \delta\left(7, Z^{6}\right) \in \Omega_{13}$, we have $\mathbf{R} \boldsymbol{P} \delta\left(7,2, Y_{12}\right)=N X_{5}+$ Fib.

This completes the proof of Proposition 2.13 for the case where $k>0$.

(ii) Suppose $k=0$ (recall that $Y_{0}=$ one-point space). By a simple $s$-number computation we see that $\mathbf{R} P \boldsymbol{\delta}\left(2^{r+2} s-1,2^{r+1}, Y_{2 k}\right)$ is indecomposable if and only if $k=0$. If we apply Corollary 2.10 and case (i) to the indecomposable $X_{2^{r+1}(2 s-1)+1}$ we have

$$
X_{2^{r+1}(2 s-1)+1}=\mathbf{R} P \boldsymbol{\delta}\left(2^{r+2} s-1,2^{r+1}, Y_{0}\right)+\sum N X_{5}^{q} X_{11}^{t}+\text { Fib. }
$$

Since every $X_{2 l+1}$ fibers over $S^{4}$ except for $X_{5}$ and $X_{11}$, the result follows and Proposition 2.13 is proven.

Referring back to Corollary 2.10 again, we now have

$$
\text { If } \omega \in \text { Tor } \Omega_{n} \text {, then } \omega=\sum N X_{5}^{q} X_{11}^{t}+\text { Fib. where } q, t \geqslant 0 \text {. }
$$

Thus all that remains to be shown to prove Theorem 2.1 is that the three oriented classes $X_{5}^{4}, X_{11}^{2}$ and $X_{5}^{2} X_{11}$ fiber over $S^{4}$. (It is known that the corresponding unoriented classes fiber over $S^{4}$, but that does not help here.)

LEMMA 2.15. The classes $X_{5}^{4} \in \Omega_{20}$ and $X_{11}^{2} \in \Omega_{22}$ fiber over $S^{4}$.

Proof. In [10] it is shown that there is a complex class $G_{5} \in \Omega_{10}^{U}$ with the property that $G_{5}^{2}$ fibers over $S^{4}$. But since $G_{5}$ is complex, $G_{5}=X_{5}^{2}$ and so $X_{5}^{4}$ is oriented cobordant to some bundle over $S^{4}$. 
By [4, Proposition 6.4] $X_{11}^{2}$ is unoriented cobordant to an orientable manifold fibered over $S^{4}$. But for dimensional reasons this is an oriented cobordism as well and so Lemma 2.15 is proven.

LEMMA 2.16. The class $X_{5}^{2} X_{11} \in \Omega_{21}$ fibers over $S^{4}$.

Proof. Let

$$
\begin{gathered}
M_{1}^{21}=\mathbf{R} P\left(\eta \oplus \theta^{13}\right) \\
\downarrow \\
\mathbf{R} P\left(\gamma \oplus \theta^{1}\right) \\
\downarrow \\
S^{4}
\end{gathered}
$$

and let

$$
\begin{gathered}
M_{2}^{21}=\mathbf{R} P\left(\eta \oplus \theta^{7}\right) \\
\downarrow \\
\mathbf{R} P\left(\gamma \oplus \theta^{7}\right) \\
\downarrow \\
S^{4} .
\end{gathered}
$$

These manifolds are orientable $\left(w_{1}\left(M_{i}\right)=0\right)$ and being odd dimensional, represent torsion classes in $\Omega_{*}$. Thus using Corollary 2.10 and Proposition 2.13 we have

$$
M_{i}=a_{i} \mathbf{R} P \delta\left(19,2, Y_{0}\right)+b_{i} \mathbf{R} P \delta\left(7,2, Y_{12}\right)+c_{i} X_{5}^{2} X_{11}+N X_{5}+\text { Fib. }
$$

The coefficients $a_{i}, b_{i}$ and $c_{i}$ can be computed via the $s$-numbers $s_{21}, s_{11,10}$ and $s_{11,5.5}$, each of which vanishes on the classes included in $N X_{5}+$ Fib. For $\mathbf{R} P \delta\left(19,2, Y_{0}\right)$ we have $s_{21}=s_{11,10}=1$ and $s_{11,5,5}=0$. For $\operatorname{RP} \delta\left(7,2, Y_{12}\right)$ we have $s_{21}=0, s_{11,10}=1$ and $s_{11,5,5}=0$. Meanwhile, $s_{21}\left(M_{1}\right)=1, s_{11,10}\left(M_{1}\right)=0, s_{11,5,5}\left(M_{1}\right)=0$ and $s_{21}\left(M_{2}\right)$ $=1, s_{11,10}\left(M_{2}\right)=0, s_{11,5,5}\left(M_{2}\right)=1$. Accordingly $a_{i}=b_{i}=1$ while $c_{1}=0$ but $c_{2}=$ 1. If we add $M_{1}$ and $M_{2}$ as given in (2.17), we get that $X_{5}^{2} X_{11}=N X_{5}+$ Fib. Finally, if we apply the De Rham invariant $w_{2} w_{19}$ (which vanishes on $X_{5}^{2} X_{11}$ as well as on any manifold fibered over $\left.S^{4}\right)$ we get that $w_{2} w_{19}\left(N X_{5}\right)=0$ and therefore $w_{16}\left(N^{16}\right)=0$ (since $w_{2} w_{3}\left(X_{5}\right) \neq 0$ ).

(2.18) Claim. If $N^{n}, n \equiv 0(\bmod 4)$, is complex with $w_{n}\left(N^{n}\right)=0$, then, for any class $T \in$ Tor $\Omega_{*}$, the class $N T \in \Omega_{*}$ fibers over $S^{4}$.

Proof. If $w_{n}\left(N^{n}\right)=0$, then $\sigma\left(N^{n}\right)$ is even and so $N=N_{0}+2 \bar{N} \in \Omega_{n}^{U}$ for some complex classes $N_{0}$ and $\bar{N}$ with $\sigma\left(N_{0}\right)=0$. Then $N T=N_{0} T \in \Omega_{*}$. By [10, Theorem 1], if $n \neq 8$, then $N_{0}$ fibers over $S^{4}$ and so $N T$ fibers over $S^{4}$. If $n=8$, then $3 N_{0}$ fibers over $S^{4}$ and so $N_{0} T=3 N_{0} T-2 N_{0} T$ fibers over $S^{4}$ again. This proves the claim and Lemma 2.16 .

The proof of Theorem 2.1 is now complete. We simply apply Lemmas 2.15 and 2.16 to $(2.14)$.

3. Proof of Theorem 1. $\Rightarrow$ We prove the necessity of the conditions given in (1.1) (see Remark 1.2). The first condition that the signature be zero is trivial since we 
have a fibration over a simply-connected base space. That conditions (b) and (c) are necessary was essentially proven in [11 and 4], respectively. Briefly, given a fibration $p: M^{n} \rightarrow S^{4}$ with fiber $F^{n-4}$ there is a $(\bmod 2)$ exact sequence (essentially the (mod 2) Wang sequence)

$$
\cdots \rightarrow H^{q-4}\left(F^{n-4}\right) \otimes H^{4}\left(D^{4}, S^{3}\right) \stackrel{j^{*}}{\rightarrow} H^{q}\left(M^{n}\right) \stackrel{i^{*}}{\rightarrow} H^{q}\left(F^{n-4}\right) \rightarrow \cdots,
$$

where $D^{4}$ denotes the 4-disc. Let $u \in I_{n-4}(\mathrm{SO}, 2)$. Then $i^{*} \tau_{M}^{*} u=\tau_{F}^{*} u=0$ and therefore $\tau_{M}^{*} u=j^{*}(x \otimes a)$ for some $x \in H^{q-4}(F)$ and $a \in H^{4}\left(D^{4}, S^{3}\right)=\mathbf{Z}_{2}$. Conditions (b) and (c) follow from the fact that the map $j^{*}$ preserves cup products and commutes with the Steenrod squares.

Condition $(d)$ arises from a $(\bmod 3)$ Wang sequence. The details are given in [10, p. 309].

As indicated in Remark 1.2, all that actually remains to be shown is the following Claim. Sq $v_{2 k-2} \in I_{4 k-4}(\mathrm{SO}, 2)$.

Proof. Let $F$ be an orientable $4 k-4$ manifold and, let $x \in H^{2 k-3}\left(F^{4 k-4} ; \mathbf{Z}_{2}\right)$. Then

$$
\begin{aligned}
\left\langle\mathrm{Sq}^{1} v_{2 k-2} \cup x,[F]\right\rangle & =\left\langle v_{2 k-2} \cup \mathrm{Sq}^{1} x,[F]\right\rangle \quad \text { (since } F \text { is orientable) } \\
& =\left\langle\mathrm{Sq}^{1} x \cup \mathrm{Sq}^{1} x,[F]\right\rangle \\
& =\left\langle x \cup \mathrm{Sq}^{1} \mathrm{Sq}^{1} x,[F]\right\rangle \quad \text { (since } F \text { is orientable) } \\
& =0 \quad \text { (by the Adem relations). }
\end{aligned}
$$

Therefore $\operatorname{Sq}^{1} v_{2 k-2}(F)=0$. This proves the claim and in turn the necessary part of Theorem 1.

$\Leftarrow$ (i) Let $\omega \in \Omega_{8}^{\mathrm{SO}}$. Then $\omega=a_{1} \mathbf{C P}(2)^{2}+a_{2} \mathbf{C P}(4), a_{i} \in \mathbf{Z}$. Since $\sigma(\omega)=0$, $a_{2}=-a_{1}$ and with $p_{1}^{2}(\omega)=3 l$ for some $l \in Z$, we get $a_{1} p_{1}^{2}\left[C P(2)^{2}-\mathbf{C P}(4)\right]=3 l$. So $a_{1}=-3 l$. But $3\left(\mathbf{C P}(2)^{2}-\mathbf{C P}(4)\right) \in \Omega_{8}^{U}$ fibers over $S^{4}[10]$. Hence $\omega=$ $-3 l(C P(2))^{2}-(C P(4))$ fibers over $S^{4}$.

(ii) Let $\omega \in \Omega_{4 k}, 4 k \neq 8$. We can write $\omega=U+T$, where $U$ is a complex manifold and $T$ denotes a torsion class. Now $\sigma(\omega)=0$ implies that $\sigma(U)=0$ and so $U$ fibers over $S^{4}$ [10, Theorem 1]. By Theorem 2.1 then, $\omega=N X_{5} X_{11}+$ Fib. Applying $\mathrm{Sq}^{2} \mathrm{Sq}^{1} v_{2 k-2} \mathrm{Sq}^{1} v_{2 k-2}$ to both sides gives $\mathrm{Sq}^{2} \mathrm{Sq}^{1} v_{2 k-2} \mathrm{Sq}^{1} v_{2 k-2}\left(N X_{5} X_{11}\right)=$ 0 . Using the product formula for characteristic classes together with the fact that $\mathrm{Sq}^{2} \mathrm{Sq}^{1} v_{6} \operatorname{Sq}^{1} v_{6}\left(X_{5} X_{11}\right) \neq 0$, we see that $w_{4 k-16}\left(N^{4 k-16}\right)=0$. That $\omega$ fibers over $S^{4}$ follows from (2.18).

(iii), (iv), (v) Let $\omega \in \Omega_{4 k+i}$, where $i=1,2$ or 3 . Then $\omega \in$ Tor $\Omega_{*}$ and so by Theorem 2.1, $\omega=N X_{5}+$ Fib., $N X_{5}^{2}+$ Fib. or $N_{1} X_{11}+N_{2} X_{5}^{3}+$ Fib., respectively. As in (ii) if we apply the appropriate numbers $\left(w_{2} w_{4 k-1}, w_{4} w_{4 k-2}\right.$ or $w_{2}^{3} \mathrm{Sq}^{2 k-3} v_{2 k}$ and $w_{6} \mathrm{Sq}^{2 k-3} v_{2 k}$, respectively) we get that in each case $w_{n}\left(N^{n}\right)=0$. Once again invoking (2.18), $\omega$ fibers over $S^{4}$.

4. Fiberings over products of surfaces. The general problem of determining which oriented cobordism classes $\omega \in \Omega_{*}$ fiber over a given $n$-manifold $M^{n}$ is very much related to the problem of determining the fibering ideal of the $n$-sphere $S^{n}$. In [16] 
Stong observed that if $\alpha \in \mathfrak{N}_{*}$ fibers over $M^{\prime}$ and $f: M \rightarrow M^{\prime}$ is bordant to the identity, then $\alpha$ fibers over $M$. He noted that consequently, if a class $\alpha \in \mathfrak{R}_{*}$ fibers over $S^{n}$, it fibers over any manifold $M^{q}$ with $q \leqslant n$. The analogous results in the context of oriented bordism (considering only orientable manifolds) also hold. The arguments are the same. But in fact, since every nonorientable manifold has an orientable double cover, we actually have:

(4.1) If an oriented class $\omega \in \Omega_{*}$ fibers over $S^{n}$, then $\omega$ fibers over any (orientable or not) manifold $M^{q}, q \leqslant n$.

In addition, if we consider products of maps oriented bordant to the identity, we get:

(4.2) If a class $\omega \in \Omega_{*}$ fibers over $S^{2} \times S^{2}$, then $\omega$ fibers over any product $B_{1}^{2} \times B_{2}^{2}$, where $B_{1}^{2}$ and $B_{2}^{2}$ are surfaces.

Indeed, if we refer to the more general "pullback" results of [1, pp. 267-268] and take product maps as before, we get:

(4.3) If a class $\omega \in \Omega_{*}$ fibers over $B_{1}^{2} \times B_{2}^{2}$, then $\omega$ fibers over $B_{1}^{2} \times B_{2}^{2} \# T$, where $B_{1}$ and $B_{2}$ are arbitrary surfaces and $T=S^{1} \times S^{1}$ denotes the torus.

We now turn to the proof of Theorem 2.

Proof. Case 1. Suppose that $\chi\left(B_{1}\right)$ or $\chi\left(B_{2}\right)$ is even.

$\Rightarrow$ Necessity follows simply by applying [1, Theorem 3$]$ to the composition of the given fibration over $B_{1} \times B_{2}$ with the appropriate projection map onto $B_{1}$ or $B_{2}$.

$\leftarrow$ To show sufficiency, we consider fibrations over $S^{2} \times S^{2}$.

LEMMA 4.4. Let $\omega \in$ Tor $\Omega_{n}$. If $n$ is even, $\omega$ fibers over $S^{2} \times S^{2}$. If $n$ is odd, then $\omega=N X_{5}+$ Fib.

Proof. We show that the classes $X_{5}^{2} \in \Omega_{10}$ and $X_{11} \in \Omega_{11}$ fiber over $S^{2} \times S^{2}$. That $X_{5}^{2}$ fibers over $S^{2} \times S^{2}$ follows from the fact [10, p. 315] that the complex class $G_{5}+C P(1) C P(2)^{2} \in \Omega_{10}^{U}$ fibers over $S^{2} \times S^{2}, G_{5}$ denoting an indecomposable. Reducing to oriented bordism shows that $G_{5} \in \Omega_{10}$ fibers over $S^{2} \times S^{2}$. But since $G_{5}$ is complex, it must be unoriented cobordant and so oriented cobordant to $X_{5}^{2}$.

For $X_{11}$, consider the iterated projective bundle

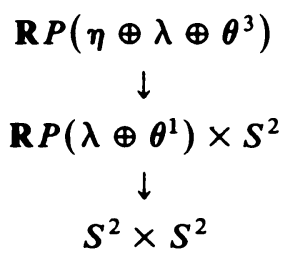

where $\lambda$ is the canonical complex line bundle over $S^{2}=C P(1)$ and $\eta$ is the canonical real line bundle over $\operatorname{R} P\left(\lambda \oplus \theta^{1}\right)$. The standard check of $s$-numbers shows that $s_{11}$ is nonzero and so this bundle (which is orientable since $w_{1}=0$ ) represents $X_{11}$. Thus $X_{11}$ fibers over $S^{2} \times S^{2}$.

The lemma now follows from Theorem 2.1 and (4.1).

Continuing the proof of Case 1 , we note as in the proof of Theorem 1 , any class $\omega \in \Omega_{4 k}$ can be written as $\omega=U+T$. Combining the fact that any complex class $U \in \Omega_{4 k}^{U}$ with $\sigma(U)=0$ fibers over $S^{2} \times S^{2}[10$, Theorem 2], with Lemma 4.4 
proves the result for fiberings over $S^{2} \times S^{2}$. Appealing to (4.2) completes the proof.

Case 2. By the classification of surfaces, we are left to consider fiberings over $B \times \mathbf{R} P(2)$.

$\Rightarrow$ The necessary condition that $\sigma(\omega)=0$ is once again trivial. Simply project to $\mathbf{R} P(2)$.

$\leftarrow$ Let

$$
\begin{aligned}
M^{9}= & \mathbf{R} P\left(\eta \oplus \lambda \oplus \theta^{2}\right) \\
\downarrow & \mathbf{R} P\left(\lambda \oplus \theta^{2}\right) \times \mathbf{R} P(2) \\
\downarrow & \mathbf{R} P(2) \times \mathbf{R} P(2)
\end{aligned}
$$

where $\lambda$ and $\eta$ are the appropriate canonical line bundles. A routine check of Stiefel-Whitney numbers shows that $w_{2} w_{7}\left[M^{9}\right]=1$. Since $M^{9}$ is orientable $\left(w_{1}(M)\right.$ $=0$ ), we know that $M^{9}=a_{1} X_{9}+a_{2} C P(2) X_{5} \in \Omega_{9}$. But $X_{9}$ fibers over $S^{4}$, so $w_{2} w_{7}\left[X_{9}\right]=0$ and hence $a_{2}=1$. Then $C P(2) X_{5}=M^{9}+a_{1} X_{9}$ and so, using (4.1), $\mathbf{C} P(2) X_{5}$ fibers over $\mathbf{R} P(2) \times \mathbf{R} P(2)$. We claim further that $\mathbf{C} P\left(2^{i}\right) X_{5}$ fibers over $\mathbf{R} P(2) \times \mathbf{R} P(2)$. This is because, as stated above, any class $\omega \in \Omega_{4 k}^{U}$ with $\sigma(\omega)=0$ fibers over $S^{2} \times S^{2}$ and hence over $\mathbf{R} P(2) \times \mathbf{R} P(2)$. Thus $\left(\mathbf{C} P\left(2^{i}\right)-\mathbf{C P}(2)^{2^{i-1}}\right) X_{5}$ fibers over $\mathbf{R} P(2) \times \mathbf{R} P(2)$ and consequently, so does $\mathbf{C P}\left(2^{i}\right) X_{5}$.

If we turn now to Lemma 4.4 , we see that any class $\omega \in$ Tor $\Omega_{*}^{\text {SO }}\left(\omega \neq X_{5}\right)$ fibers over $\mathbf{R} P(2) \times \mathbf{R} P(2)$. The "splitting" $\Omega_{4 k}=U+T$ then gives Theorem 2 for $\mathbf{R} P(2) \times \mathbf{R} P(2)$ and invoking (4.3) completes Case 2 entirely.

\section{REFERENCES}

1. J. C. Alexander and S. M. Kahn, Characteristic number obstructions to fibering oriented and complex manifolds over surfaces, Topology 19 (1980), 265-282.

2. P. G. Anderson, Cobordism classes of squares of orientable manifolds, Ann. of Math. (2) 83 (1966), 47-53.

3. E. H. Brown, Jr. and F. P. Peterson, Relations among characteristic classes. I, Topology 3 (1964), suppl. 1, 39-52.

4. R. L. W. Brown, Cobordism and bundles over spheres, Michigan Math. J. 16 (1969), 315-320.

5. R. O. Burdick, Oriented manifolds fibered over the circle, Proc. Amer. Math. Soc. 17 (1966), 449-452.

6. D. Chess, Cobordism and the nonfinite homotopy type of some diffeomorphism groups, preprint, 1983.

7. P. E. Conner, The bordism class of a bundle space, Michigan Math. J. 14 (1967), 289-303.

8. P. E. Conner and E. E. Floyd, Fibering within a cobordism class, Michigan Math. J. 12 (1965), $33-47$.

9. A. Didierjean, Classes de cohomologie invariantes par cobordisme et classes de cobordisme se fibrant sur des spheres, C. R. Acad. Sci. Paris 289 (1979), 165-167.

10. S. M. Kahn, Fibering complex manifolds over $S^{3}$ and $S^{4}$, Illinois J. Math. 26 (1982), 296-316.

11. Cobordism obstructions to fibering manifolds over spheres, Pacific J. Math. (to appear).

12. M. Kreck, Cobordism of odd-dimensional diffeomorphisms, Topology 15 (1976), 353-361.

13. W. Meyer, Die signature von flachenbundlen, Math. Ann. 201 (1973), 239-264.

14. W. D. Neumann, Fibering over a circle within a cobordism class, Math. Ann. 192 (1971), 191-192.

15. D. F. X. O'Reilly, Cobordism classes of fiber bundles, Pacific J. Math. 69 (1977), 467-475.

16. R. E. Stong, On fibering of cobordism classes, Trans. Amer. Math. Soc. 178 (1973), 431-447.

17. C. T. C. Wall, Determination of the cobordism ring, Ann. of Math. (2) 72 (1960), 292-311.

Department of Mathematics, Wayne State University, Detroit, Michigan 48202 